\title{
Optical evidence for the proximity to a spin-density-wave metallic state in $\mathrm{Na}_{0.7} \mathrm{CoO}_{2}$
}

\author{
G. Caimi and L. Degiorgi \\ Laboratorium für Festkörperphysik, ETH Zürich, CH-8093 Zürich, Switzerland \\ H. Berger, N. Barisic, and L. Forró \\ Institut de physique de la matière complexe (IPMC), \\ EPF Lausanne, CH-1015 Lausanne, Switzerland \\ F. Bussy \\ Institute of Mineralogy and Geochemistry, \\ University of Lausanne, CH-1015 Lausanne, Switzerland
}

(Dated: November 23, 2018)

\begin{abstract}
We present the optical properties of $\mathrm{Na}_{0.7} \mathrm{CoO}_{2}$ single crystals, measured over a broad spectral range as a function of temperature $(T)$. The capability to cover the energy range from the farinfrared up to the ultraviolet allows us to perform reliable Kramers-Kronig transformation, in order to obtain the absorption spectrum (i.e., the complex optical conductivity). To the complex optical conductivity we apply the generalized Drude model, extracting the frequency dependence of the scattering rate $(\Gamma)$ and effective mass $\left(m^{*}\right)$ of the itinerant charge carriers. We find that $\Gamma(\omega) \sim \omega$ at low temperatures and for $\omega>T$. This suggests that $\mathrm{Na}_{0.7} \mathrm{CoO}_{2}$ is at the verge of a spin-density-wave metallic phase.

PACS numbers: 78.20.-e, 74.70.Dd, 75.30.Fv
\end{abstract}


The discovery of superconductivity at $5 \mathrm{~K}$ in hydrated sodium cobaltate[1] has attracted considerable attention. How water inclusion triggers superconductivity in $\mathrm{Na}_{x} \mathrm{CoO}_{2}$ is not fully understood yet. The investigation of non-hydrated sample is therefore of relevance and a considerable research effort has been devoted to $\mathrm{Na}_{x} \mathrm{CoO}_{2}$ specimens with $x$ ranging between 0.3 and 0.75 . As $x$ increases from 0.3 , the ground state goes from a paramagnetic metal to a charge-ordered insulator for $x=0.5$ to a Curie-Weiss metal around 0.7 and finally to a weak-moment magnetically ordered state for $x>0.75$ (Ref. 2). This latter phase is supposed to be equivalent to a so called spin-density-wave (SDW) metal. Several recent investigations $[3,4,5]$, based on magnetic, thermal and transport properties as well as muon spin spectroscopy, indicate the formation of a SDW metallic state for $x=0.75$.

Optical experiments are well-known tools in order to achieve information about the electrodynamic response of the investigated system, and to shed light on its electronic structure. From the absorption spectrum one can also extract the effective mass and the scattering rate of the itinerant charge carriers. Lupi et al. measured the optical conductivity of $\mathrm{Na}_{x} \mathrm{CoO}_{2}$ (for $x=0.57$ ) and reported an "anomalous Drude" behavior, where the charge carrier have an effective mass of $5 m_{b}$ and their scattering rate is $\Gamma(\omega)=\frac{1}{\tau(\omega)} \sim \omega^{3 / 2}$ (Ref. 6). Wang et al. optically investigated $\mathrm{Na}_{0.7} \mathrm{CoO}_{2}$, mainly focusing their attention on the high frequency spectral range. They found two broad interband transitions at $1.6 \mathrm{eV}$ and $3.2 \mathrm{eV}$ ascribed to the $t_{2 g}-e_{g}$ band transitions by invoking the effect of the exchange splitting[7]. Additionally, a mid-infrared peak at about $0.4 \mathrm{eV}$ was attributed to interband transition within the $t_{2 g}$ manifold or to the electronic correlation effect. At lower frequencies a non simple Drude intraband response was detected, as well[7].

In this short communication, we offer our optical investigations on high quality $\mathrm{Na}_{0.7} \mathrm{CoO}_{2}$ single crystals. Our specimens have a size 2 × $2 \mathrm{~mm}$ and were grown by the flux methods as thoroughly described elsewhere[8]. Samples from the same batch were furthermore characterized by the dc transport measurements[9]. The temperature dependence of the resistivity $\rho(T)$ (not shown here) within the $a b$ plane displays a linear behavior in temperature from 300 down to $100 \mathrm{~K}$, as well as below $100 \mathrm{~K}$, however with a smaller slope, in agreement with data of Ref. 2 and 10. We performed optical reflectivity measurements from the far-infrared (FIR) up to the ultraviolet (i.e., $5 \mathrm{meV}-12.4 \mathrm{eV}$ ) as a function of temperature between 10 and $300 \mathrm{~K}$. Measurements were performed also in a magnetic field up to 7 T. No changes in the spectra were however found as a function of field. Our inves- 
tigations covers the largest spectral range addressed so far on $\mathrm{Na}_{x} \mathrm{CoO}_{2}$. Details pertaining the experiments can be found elsewhere[11, 12].

Figure 1 displays in the top panel the optical reflectivity $R(\omega)$. Beside some absorption at about and above $1 \mathrm{eV}$, one can recognize the quite sharp plasma edge feature with onset at $\simeq 0.7 \mathrm{eV} . \mathrm{R}(\omega)$ increases with decreasing temperature below $0.2 \mathrm{eV}$, indicative for the metallic character of $\mathrm{Na}_{0.7} \mathrm{CoO}_{2}$. Furthermore, we clearly see at $0.07 \mathrm{eV}$ the infrared-active phonon mode. Our data bears an overall similarity with the finding of Lupi et al. for $\mathrm{Na}_{0.57} \mathrm{CoO}_{2}$ (Ref. 6). There is also a rough agreement with the optical response measured on $\mathrm{Na}_{0.7} \mathrm{CoO}_{2}$ by Wang et al.[7] In this latter data, there is a crossing of the $\mathrm{R}(\omega)$ spectra around $0.1 \mathrm{eV}$ and the formation of a bump at about $0.03 \mathrm{eV}$ with decreasing temperature, for which neither our spectra nor those of Lupi et al. give a clear cut evidence.

The bottom panel of Fig. 1 shows the real part $\sigma_{1}(\omega)$ of the optical conductivity. We have extrapolated the $\mathrm{R}(\omega)$ spectra towards zero energies with the Hagen-Rubens extrapolation[11, 12], using dc values of the conductivity in agreement with the transport results $[2,5,10]$. Standard extrapolation of $R(\omega) \sim \omega^{-s}$ (with $2<s<4$ ) were employed at high frequencies. It is easy verified that the main conclusions of our work are fully independent from the employed extrapolations due to the extremely broad measured spectral range. As expected from the $R(\omega)$ spectra, the effective intraband metallic component in $\sigma_{1}(\omega)$ is enhanced below $0.03 \mathrm{eV}$ and the $\sigma_{1}(\omega \rightarrow 0)$ limit increases with decreasing temperature, as typical for a metallic system. At higher frequencies we recognize a weak feature at $0.4 \mathrm{eV}$ (A) followed by more pronounced and well defined features at $1.4 \mathrm{eV}(\mathrm{B}$,$) at 2.8 \mathrm{eV}(\mathrm{C})$, at $4.8 \mathrm{eV}(\mathrm{D})$, and at $10 \mathrm{eV}(\mathrm{E})$. These latter absorptions are in fair agreement with the data of Wang et al.[7] and in good accord with the recent findings obtained with the ellipsometry method[13]. Furthermore, angle resolved photoemission results identify electronic excitations at $0.7,3,4.1,6$ and $11 \mathrm{eV}$ (Ref. 14). In passing, we note that some of the detected absorptions could be ascribed to electronic interband transitions involving the Co $3 d t_{2 g}$ and $e_{g}$ manifolds and their exchange splitting[15, 16].

The metallic component of $\sigma_{1}(\omega)$ cannot be fully reproduced by a simple Drude term, the most common description for simple metals, also successfully applied in several oxides. The optical conductivity can be alternatively described in terms of an "anomalous or generalized Drude" model, where both the effective mass $m^{*}(\omega)$ and the scattering rate $\Gamma(\omega)$ of the itinerant charge carriers are allowed to depend on frequency. We analyze the complex 
optical conductivity $\tilde{\sigma}(\omega)=\sigma_{1}(\omega)+i \sigma_{2}(\omega)$ by applying the following expression:

$$
\tilde{\sigma}(\omega)=\frac{\omega_{p}^{2}}{4 \pi} \frac{1}{\Gamma(\omega)-i \omega \frac{m^{*}(\omega)}{m_{b}}} .
$$

By inverting eq. (1) we obtain:

$$
\begin{aligned}
\Gamma(\omega) & =\frac{\omega_{p}^{2}}{4 \pi} \frac{\sigma_{1}}{|\sigma|^{2}} \\
\frac{m^{*}(\omega)}{m_{b}} & =\frac{\omega_{p}^{2}}{4 \pi} \frac{\sigma_{2}}{\omega|\sigma|^{2}}
\end{aligned}
$$

In practice, we use $\tilde{\sigma}(\omega)$, obtained by the Kramers-Kronig analysis, in order to evaluate the frequency dependence of the effective mass and scattering rate. $\omega_{p}^{2}$ is the spectral weight associated with the itinerant charge carriers, and it can be estimated by integrating $\sigma_{1}(\omega)$ from zero frequency up to a cut-off frequency $\omega_{c}$ coinciding with onset of electronic interband transitions. We choose $\omega_{c} \approx 0.63 \mathrm{eV}$, giving a value of $\omega_{p} \simeq 1.17 \mathrm{eV}$. Figure 2 displays the frequency dependence of the scattering rate $\Gamma$ below $0.2 \mathrm{eV}$. For clarity, the depletion of $\Gamma(\omega)$ at $0.07 \mathrm{eV}$, due to the phonon mode, has been removed from the data (see below). We remark moreover that $m^{*}(\omega)$ (not shown here) weakly increases with decreasing frequency at all temperatures, reaching a value of about $5 m_{b}$ in the limit $\omega \rightarrow 0$. This agrees with Lupi's data[6].

As expected, $\Gamma(\omega)$ decreases with decreasing temperature. Over a very broad spectral range, extending up to about $0.15 \mathrm{eV}, \Gamma(\omega)$ can be fitted with the power law expression:

$$
\Gamma(\omega) \sim \omega^{\alpha}
$$

We establish that $\alpha \simeq 1$ for temperatures below about $50 \mathrm{~K} . \Gamma(\omega) \sim \omega$ might be indicative of a non-Fermi liquid behavior in $\mathrm{Na}_{0.7} \mathrm{CoO}_{2}$. The exponent $\alpha$ (Fig. 3a) tends nevertheless to decrease at higher temperatures (e.g., $\alpha=0.75$ at $200 \mathrm{~K}$ ). We emphasize at this point that our fit after eq. (4) is not affected by the ad-hoc phonon subtraction. The inset of Fig. 2 shows indeed that even the original curve of $\Gamma(\omega)$ at $10 \mathrm{~K}$ can be well fitted by eq. (4) with $\alpha=1$. The same applies for all temperatures, making our analysis of $\Gamma(\omega)$ robust. The linear frequency dependence of $\Gamma(\omega)$ at $\omega>T$ pairs with the linear temperature dependence of $\rho_{d c}(T)$ for $T<100 \mathrm{~K}$ in the compound with $71 \%$ Na content[2, 9].

Ruvalds and Virosztek proposed a while ago a Fermi-surface nesting scenario for describing the optical properties of superconducting oxides[17]. They showed that Fermi-surface 
nesting modifies the electron-electron scattering and therefore yields an unusual variation of the optical reflectivity. Within this scenario, also applicable for the charge- and spindensity-wave state where nesting is an essential ingredient[17], the effective Drude component is characterized by a relaxation rate that is linear in frequency for $\omega>T$ (Fig. 2). Ruvalds and Virosztek[17] also predicts that $\Gamma(\omega \rightarrow 0) \sim T$, as verified in Fig. 3b, where $\Gamma(\omega)$ in the static limit $\omega \rightarrow 0$ as a function of $T$ is shown. Furthermore, $R(\omega)$ is linear in $\omega$ in a broad spectral range (inset of Fig. 1), in agreement with the theory[17]. Our findings in $\mathrm{Na}_{0.7} \mathrm{CoO}_{2}$ support the Fermi-surface nesting scenario. Consequently, considering the phase diagram reported in Ref. 2, we can then affirm that $\mathrm{Na}_{0.7} \mathrm{CoO}_{2}$ seems to be at the verge of a SDW metallic phase. The linear frequency dependence of $\Gamma(\omega)$ at low temperatures differs from the conclusion of Lupi et al. on $\mathrm{Na}_{0.57} \mathrm{CoO}_{2}$, for which $\alpha \simeq 3 / 2$ (Ref. 6). This could be explained by the different stoichiometry of the samples. $\mathrm{Na}_{0.57} \mathrm{CoO}_{2}$ is quite close to the charge-ordered insulating boundary (at $x=0.5$ ) between a paramagnetic metal and a Curie-Weiss metal[2]. On the contrary, our sample $\mathrm{Na}_{0.7} \mathrm{CoO}_{2}$ is located in the phase diagram[2] well within the Curie-Weiss metal sector and is close to the boundary (at least for low temperatures) of the SDW metallic phase. We shall finally note that after Ref. 17 a non Fermi liquid-Fermi liquid crossover is not excluded at very low temperatures in the case of an imperfect Fermi-surface nesting. This would be in agreement with the dc-transport data on $\mathrm{Na}_{0.7} \mathrm{CoO}_{2}$. Indeed for $T<1 \mathrm{~K}, \rho(T)$ displays a typical Fermi liquid $T^{2}$ behavior[18].

In conclusion, we have provided the complete absorption spectrum of $\mathrm{Na}_{0.7} \mathrm{CoO}_{2}$. The frequency dependence of the scattering rate of the itinerant charge carriers is extracted from the complex optical conductivity, and we have established that $\Gamma(\omega) \sim \omega$ at low temperatures. $\mathrm{Na}_{0.7} \mathrm{CoO}_{2}$ seems to be in the proximity of a spin-density-wave metallic state. It turns out[2] that the exact stoichiometry plays an essential role in defining the intrinsic physical properties of $\mathrm{Na}_{x} \mathrm{CoO}_{2}$. As future outlook, we wish to fine tuning the $\mathrm{Na}$ content $x$ between 0.7 and 0.85 and study the corresponding optical response. The SDW metallic phase for $x \geq 0.75$ is an appealing scenario, supported by various experimental results, but might be not the unique one. Interestingly enough, Bernhard et al.[13] found evidence for a polaronic band in the case of $x=0.82$.

The authors wish to thank J. Müller for technical help and A. Perucchi for fruitful discussions. This work has been supported by the Swiss National Foundation for the Scientific Research. 
[1] K. Takada, H. Sakurai, E. Takayama-Muromachi, F. Izumi, R. A. Dilanian, and T. Sasaki, Nature 422, 53 (2003).

[2] M. L. Foo, Y. Wang, S. Watauchi, H. W. Zandbergen, T. He, R. J. Cava, and N. P. Ong, cond-mat/0312174.

[3] B. C. Sales, R. Jin, K. A. Affholter, P. Khalifah, G. M. Veith, and D. Mandrus, condmat/0402379.

[4] J. Sugiyama, J. H. Brewer, E. J. Ansaldo, H. Itahara, T. Tani, M. Mikami, Y. Mori, T. Sasaki, S. Hebert, and A. Maignan, cond-mat/0310516.

[5] T. Motohashi, R. Ueda, E. Naujalis, T. Tojo, I. Terasaki, T. Atake, M. Karppinen, and H. Yamauchi, Phys. Rev. B 67, 064406 (2003).

[6] S. Lupi, M. Ortolani, and P. Calvani, cond-mat/0312512.

[7] N. L. Wang, P. Zheng, D. Wu, Y. C. Ma, T. Xiang, R. Y. Jin, and D. Mandrus, condmat/0312630.

[8] M. N. Iliev, A. P. Litvinchuk, R. L. Meng, Y. Sun, J. Cmaidalka, and C. W. Chu, Physica C 402, 239 (2004).

[9] The dc resistivity $\rho(T)$ was measured within the $a b$ plane and along the $c$-axis at EPFLausanne, with the conventional four points contact method.

[10] Y. Wang, N. S. Rogado, R. J. Cava, and N. P. Ong, Nature 423, 425 (2003).

[11] F. Wooten, Optical Properties of Solids (Academic Press, New York, 1972).

[12] M. Dressel and G. Grüner, Electrodynamics of Solids (Cambridge University Press, Cambridge, 2002).

[13] C. Bernhard, A. V. Boris, N. N. Kovaleva, G. Khaliullin, A. Pimenov, L. Yu, D. P. Chen, C. T. Lin, and B. Keimer, cond-mat/0403155.

[14] M. Z. Hasan, Y. D. Chuang, A. P. Kuprin, Y. Kong, D. Qian, Y. W. Li, B. L. Mesler, Z. Hussain, A. V. Fedorov, R. Kimmerling, E. Rotenberg, K. Rossnagel, H. Koh, N. S. Rogado, M. L. Foo, and R. J. Cava, cond-mat/0308438.

[15] D. J. Singh, Phys. Rev. B 61, 13397 (2000).

[16] J. Kunes, K. W. Lee, and W. E. Pickett, cond-mat/0308388 .

[17] J. Ruvalds and A. Virosztek, Phys. Rev. B 43, 5498 (1991). 
[18] S. Y. Li, L. Taillefer, D. G. Hawthorn, M. A. Tanatar, J. Paglione, M. Sutherland, R. W. Hill, C. H. Wang, and X. H. Chen, cond-mat/0401099. 


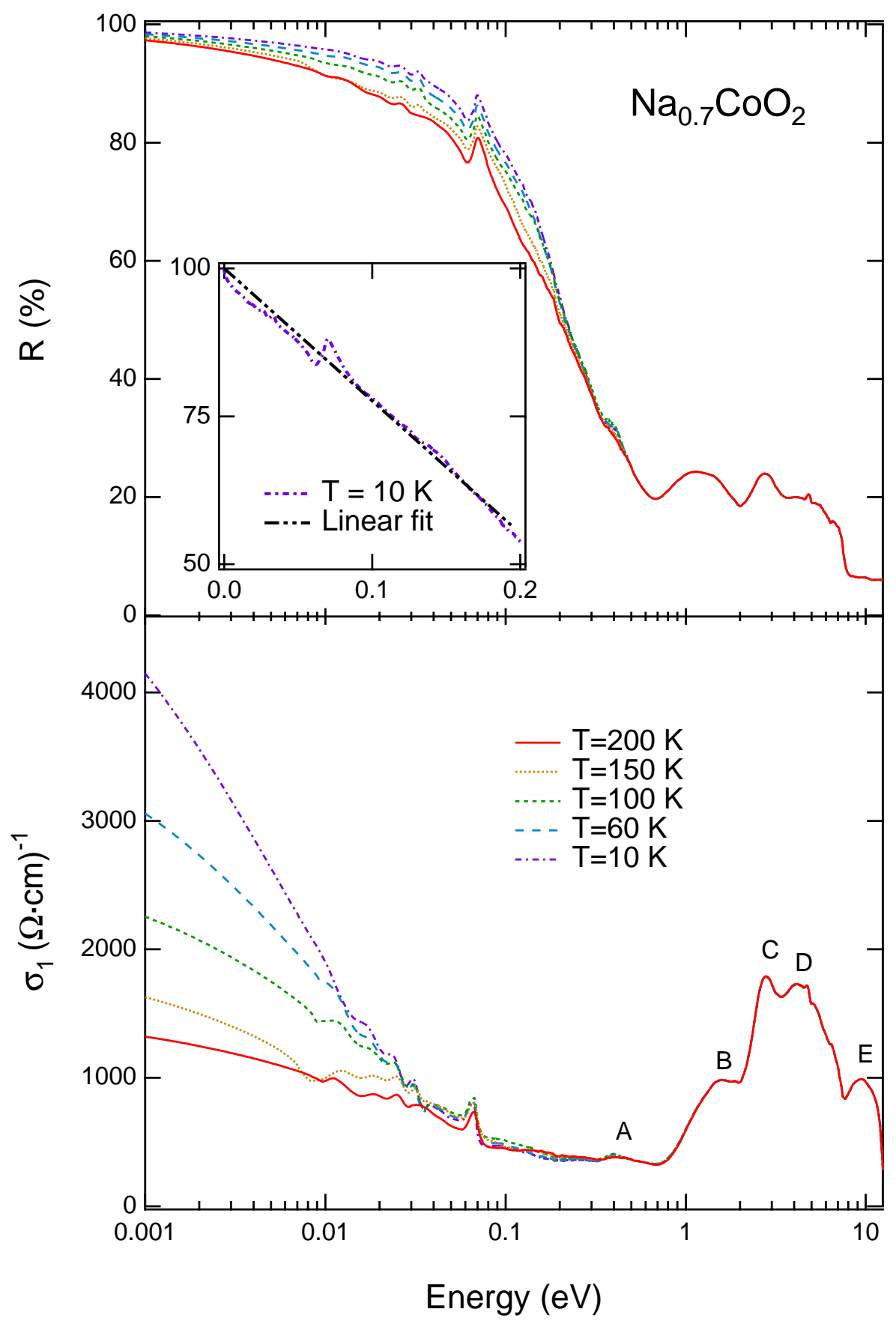

FIG. 1: (Color online) Reflectivity (top panel) and real part $\sigma_{1}(\omega)$ of the optical conductivity (bottom panel) of $\mathrm{Na}_{0.7} \mathrm{CoO}_{2}$ at selected temperatures. The four high frequency absorptions in $\sigma_{1}(\omega)$ are labeled (see text). Inset: $\mathrm{R}(\omega)$ at $10 \mathrm{~K}$ between 0 and $0.2 \mathrm{eV}$, emphasizing the linear behavior of $R(\omega)$ at low energies. 


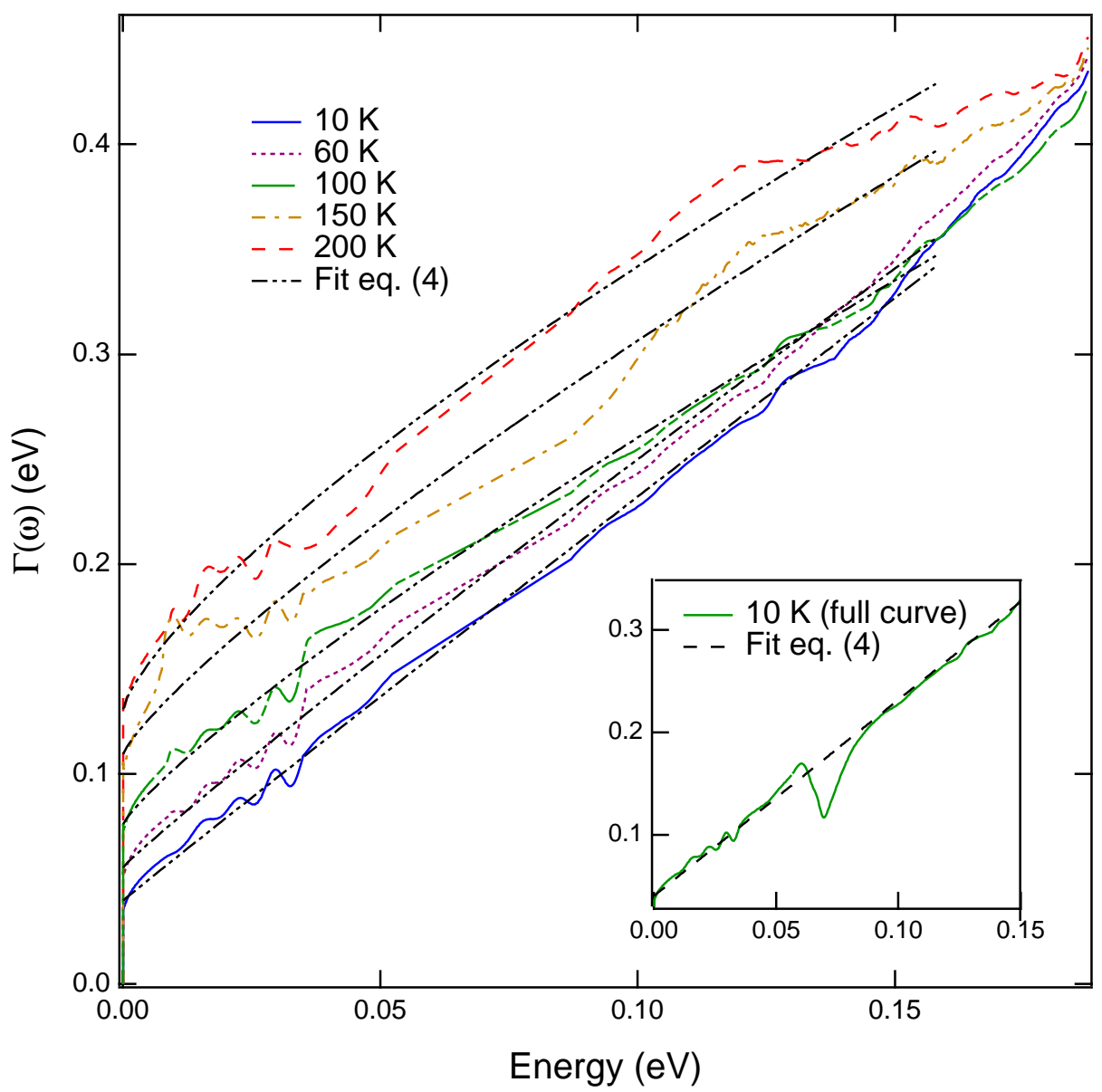

FIG. 2: (Color online) Frequency dependence of the scattering rate and its fit according to eq. (4) at selected temperatures. Note that the IR active phonon has been subtracted in order to better highlight the linear or sub-linear fit. Inset: the original curve of $\Gamma(\omega)$ (i.e., comprehensive of the IR phonon at $0.07 \mathrm{eV}$ ) at $10 \mathrm{~K}$ is shown with the fit after eq. (4) with $\alpha=1$. The phonon subtraction does not affect the fit of $\Gamma(\omega)$. This is true at all temperatures. 


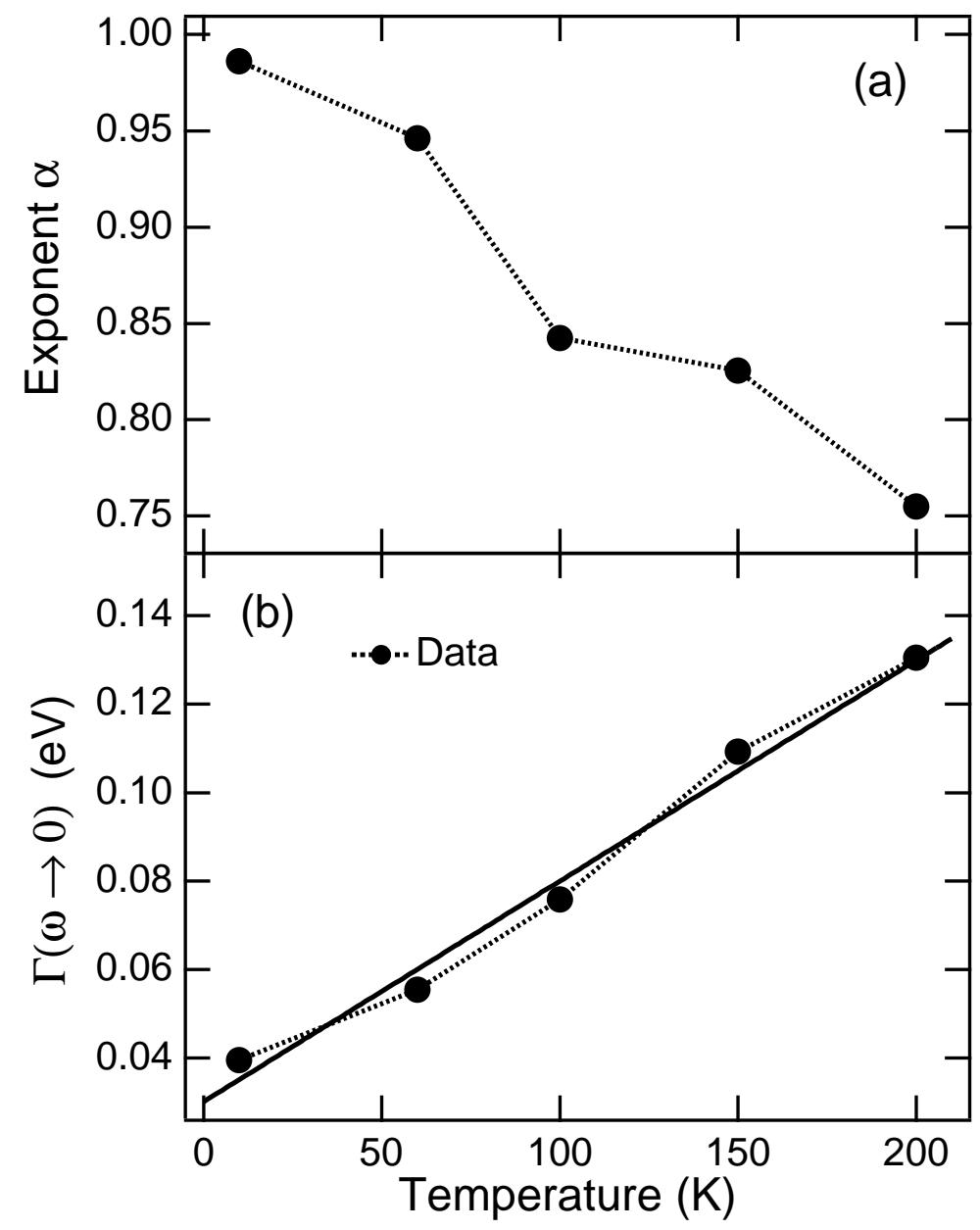

FIG. 3: (a) Temperature dependence of the exponent $\alpha$ in eq. (4). (b) Temperature dependence of $\Gamma(\omega)$ in the static limit $\omega \rightarrow 0 . \Gamma(\omega \rightarrow 0)$ can be well approximated by a linear fit. 